\title{
Generation of long-living entanglement between two separate three-level atoms
}

\author{
Özgür Çakir, ${ }^{1}$ Ho Trung Dung, ${ }^{2}$ Ludwig Knöll, ${ }^{3}$ and Dirk-Gunnar Welsch ${ }^{3}$ \\ ${ }^{1}$ Department of Physics, Bilkent University, 06533 Bilkent, Ankara, Turkey \\ ${ }^{2}$ Institute of Physics, National Center for Sciences and Technology, 1 Mac Dinh Chi Street, District 1, Ho Chi Minh City, Vietnam \\ ${ }^{3}$ Theoretisch-Physikalisches Institut, Friedrich-Schiller-Universität Jena, Max-Wien-Platz 1, 07743 Jena, Germany
}

(Received 11 October 2004; revised manuscript received 21 December 2004; published 18 March 2005)

\begin{abstract}
A scheme for nonconditional generation of long-living maximally entangled states between two spatially well separated atoms is proposed. In the scheme, $\Lambda$-type atoms pass a resonatorlike equipment of dispersing and absorbing macroscopic bodies giving rise to body-assisted electromagnetic field resonances of well-defined heights and widths. Strong atom-field coupling is combined with weak atom-field coupling to realize entanglement transfer from the dipole-allowed transitions to the dipole-forbidden transitions, whereby the entanglement is preserved when the atoms depart from the bodies and from each other. The theory is applied to the case of atoms passing by a microsphere.
\end{abstract}

DOI: 10.1103/PhysRevA.71.032326

PACS number(s): 03.67.Mn, 03.65.Ud, 42.50.Nn, 42.50.Dv

\section{INTRODUCTION}

Generation of entanglement in atomic systems has been a subject of intense theoretical and experimental study motivated by both the fundamental issue and potential applications in quantum-information processing. In this context, the realization of easily controllable long-living entangled states of spatially well-separated atoms has been one of the crucial and challenging problems. A number of methods of entanglement preparation between atoms have been considered, such as the use of quantum-correlated light fields interacting with separate atoms, thereby transferring their entanglement to the atoms [1-7], appropriate measurements on the light in multiatom-light interaction processes, thereby conditionally projecting the atoms in entangled states [8-18], and the technique of quantum reservoir engineering in a cascaded cavityQED setting [19].

Photon exchange between two atoms is one of the simplest processes to entangle two atoms in a common electromagnetic field. The effect, which is very weak in free space, can be enhanced significantly when the atoms are in a cavity [20-22]. Usually attempts are made to minimize the effect of spontaneous emission. Quite counterintuitively, in certain situations one can take advantage of the spontaneous emission for entanglement generation [23-26]. Consider, for example, two two-level atoms located in free space with one of them being initially excited. This product state is a superposition of a symmetric (superradiant) state and an antisymmetric (subradiant) state. If the two atoms are separated by distances much smaller than the wavelength, the symmetric state decays must faster than the antisymmetric one, leaving the system in a mixture of the ground state and the entangled antisymmetric state.

The scheme also works at distances much larger than the wavelength, if a resonatorlike equipment is used which sufficiently enhances the atom-field coupling, thereby ensuring that a photon emitted in the process of resonant photon exchange, which is mediated by real photon emission and absorption, is accessible to the two atoms. This condition can be satisfied, for instance, when the atoms pass by a dielectric microsphere at diametrically opposite positions [23]. If the distance of the atoms from the surface of the sphere becomes sufficiently small, then the excitation of surface-guided (SG) and whispering-gallery (WG) waves can give rise to strong collective effects, which are necessarily required to generate substantial entanglement. Needless to say, other than spherically symmetric bodies can also be used to realize a noticeable mutual coupling of the atoms.

A drawback of the use of two-level-type atoms is that the entanglement is transient. In particular, when two atoms that have become entangled with each other near a body such as a microsphere move away from it (and from each other), then they undergo ordinary spontaneous emission (in free space), which destroys the quantum coherence. Preservation of the atomic entanglement over long distances between the atoms is therefore not possible in this way.

The contradictory effects of entanglement creation and destruction typical of two-level atoms can be combined in a more refined scheme involving two three-level atoms each of $\Lambda$ type (Fig. 1), where the two lower-lying states $|1\rangle$ and $|2\rangle$ such as the ground state and a metastable state or two metastable states represent the qubits that are desired to be entangled with each other [27]. Whereas the transition $|1\rangle \leftrightarrow|3\rangle$ is strongly coupled to the field, the transition $|2\rangle \leftrightarrow|3\rangle$ is only weakly coupled to the field. Each atom is initially in the state $|1\rangle$, while the field is prepared in a single-photon state. Let us assume that due to Rabi oscillations the state $|3\rangle$ of one of the

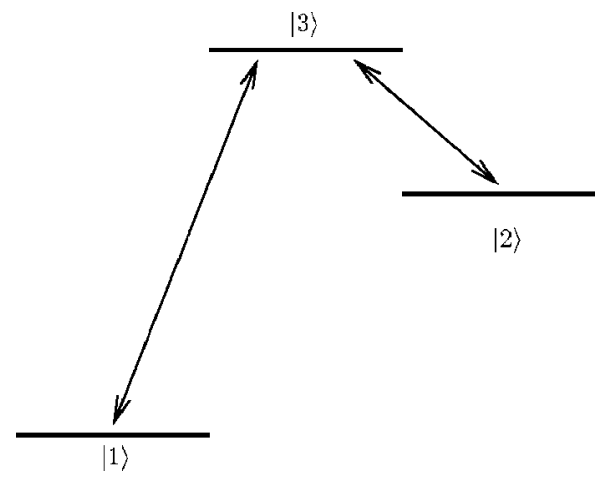

FIG. 1. Three-level atom of $\Lambda$ type. 
two atoms (we do not know which one) is populated. Irreversible decay to the state $|2\rangle$ is then accompanied by an entanglement transfer forming a (quasi)stationary entangled state between the two atoms with respect to the states $|1\rangle$ and $|2\rangle$. Its lifetime is limited only by the lifetime of the metastable states, and the degree of entanglement achievable can approach $100 \%$ in principle. Moreover, the scheme is nonconditional and realizable by means of current experimental techniques.

In fact, the model Hamiltonian used in Ref. [27] is based on a Dicke-type system and does not allow for atoms that are spatially well separated from each other, with the interatomic distance being much larger than the characteristic wavelengths. However, for many applications in quantuminformation processing or for testing Bell's inequalities, large interatomic distances and thus the possibility of individual manipulation of the atoms are necessary prerequisites. The aim of the present paper is to close this loophole, by considering two spatially well-separated $\Lambda$-type three-level atoms appropriately positioned with respect to macroscopic bodies, so that the two key ingredients-enhanced atom-field coupling and sharp field resonances can be realized. Note that the second ingredient is absent in the case of a superlens geometry [26]. To illustrate the theory, we apply it to the case of the two atoms being near a realistic dielectric microsphere. The formalism used is based on the quantization of the macroscopic electromagnetic field and allows one to take into account material dispersion and absorption in a quantum-mechanically consistent manner.

The paper is organized as follows. In Sec. II the basic equations for describing the interaction of $N$ multilevel atoms with the electromagnetic field in the presence of dispersing and absorbing macroscopic bodies are given. In Sec. III the theory is applied to the problem of formation of an entangled state between two $\Lambda$-type three-level atoms. Section IV presents the results obtained for the case when the two atoms are at diametrically opposite positions outside a microsphere. Finally, a summary and some concluding remarks are given in Sec. V.

\section{MASTER EQUATION}

Consider $N$ multilevel atoms at given positions $\mathbf{r}_{A}$ that interact with the electromagnetic field in the presence of some macroscopic, linear bodies, which are allowed to be both dispersing and absorbing. In the electric dipole approximation, the overall system can be described by the multipolar-coupling Hamiltonian [28]

$$
\begin{aligned}
\hat{H}= & \int d^{3} \mathbf{r} \int_{0}^{\infty} d \omega \hbar \omega \hat{\mathbf{f}}^{\dagger}(\mathbf{r}, \omega) \hat{\mathbf{f}}(\mathbf{r}, \omega)+\sum_{A} \sum_{m} \hbar \omega_{A m} \hat{R}_{A m m} \\
& -\sum_{A} \int_{0}^{\infty} d \omega\left[\hat{\mathbf{d}} \hat{}_{A} \underline{\hat{\mathbf{E}}}\left(\mathbf{r}_{A}, \omega\right)+\text { H.c. }\right] .
\end{aligned}
$$

Here, the bosonic fields $\hat{\mathbf{f}}(\mathbf{r}, \omega)$ and $\hat{\mathbf{f}}^{\dagger}(\mathbf{r}, \omega)$ are the canonically conjugated variables of the system, which consists of the electromagnetic field and the bodies (including the dissipative system responsible for absorption), the $\hat{R}_{A m n}$
$=|m\rangle_{A A}\langle n|$ are the atomic (flip) operators with $|m\rangle_{A}$ being the $m$ th energy eigenstate of the $A$ th atom (of energy $\hbar \omega_{A m}$ ), and $\hat{\mathbf{d}}_{A}=\Sigma_{m, n} \mathbf{d}_{A m n} \hat{R}_{A m n}$ are the electric dipole operators of the atoms $\left(\mathbf{d}_{A m n}={ }_{A}\left\langle m\left|\hat{\mathbf{d}}_{A}\right| n\right\rangle_{A}\right)$. Further, the body-assisted electric field in the $\omega$ domain, $\underline{\hat{\mathbf{E}}}(\mathbf{r}, \omega)$, expressed in terms of the fundamental variables $\hat{\mathbf{f}}(\mathbf{r}, \omega)$, reads

$$
\underline{\hat{\mathbf{E}}}(\mathbf{r}, \omega)=\int d^{3} \mathbf{r}^{\prime} \widetilde{\boldsymbol{G}}\left(\mathbf{r}, \mathbf{r}^{\prime}, \omega\right) \hat{\mathbf{f}}\left(\mathbf{r}^{\prime}, \omega\right),
$$

where

$$
\widetilde{\boldsymbol{G}}\left(\mathbf{r}, \mathbf{r}^{\prime}, \omega\right)=i \sqrt{\frac{\hbar}{\pi \varepsilon_{0}}} \frac{\omega^{2}}{c^{2}} \sqrt{\operatorname{Im} \varepsilon\left(\mathbf{r}^{\prime}, \omega\right)} \boldsymbol{G}\left(\mathbf{r}, \mathbf{r}^{\prime}, \omega\right),
$$

with $G\left(\mathbf{r}, \mathbf{r}^{\prime}, \omega\right)$ being the classical Green tensor satisfying the equation

$$
\boldsymbol{\nabla} \times \boldsymbol{\nabla} \times \boldsymbol{G}\left(\mathbf{r}, \mathbf{r}^{\prime}, \omega\right)-\frac{\omega^{2}}{c^{2}} \varepsilon(\mathbf{r}, \omega) \boldsymbol{G}\left(\mathbf{r}, \mathbf{r}^{\prime}, \omega\right)=\boldsymbol{\delta}\left(\mathbf{r}-\mathbf{r}^{\prime}\right)
$$

$[\boldsymbol{\delta}(\mathbf{r})$ is the dyadic $\delta$ function] together with the boundary conditions at infinity. Throughout the paper we restrict our attention to dielectric bodies, which are described by a spatially varying complex permittivity $\varepsilon(\mathbf{r}, \omega)=\operatorname{Re} \varepsilon(\mathbf{r}, \omega)$ $+i \operatorname{Im} \varepsilon(\mathbf{r}, \omega)$.

Next let suppose that the excitation spectrum of the bodyassisted electromagnetic field shows a resonance structure, with the lines being well separated from each other. With regard to the atom-field coupling, we assume that a few atomic transitions can be strongly coupled to field resonances tuned to them, while the other ones are weakly coupled to the field. In close analogy to Ref. [29], it can be shown that the density operator of the system consisting of the atoms and that part of the body-assisted electromagnetic field which strongly interacts with the atoms obeys the master equation

$$
\begin{aligned}
\dot{\hat{\varrho}}= & -\frac{i}{\hbar}\left[\hat{\widetilde{H}}_{S}, \hat{\varrho}\right]+\left[i \sum_{A, A^{\prime}} \sum_{m, n} \delta_{A A^{\prime}}^{m n}\left(\hat{R}_{A m n} \hat{R}_{A^{\prime} n m} \hat{\varrho}-\hat{R}_{A^{\prime} n m} \hat{\varrho} \hat{R}_{A m n}\right)\right. \\
& + \text { H.c. }]-\frac{1}{2} \sum_{A, A^{\prime}} \sum_{m, n}{ }^{\prime}\left[\Gamma_{A A^{\prime}}^{m n}\left(\hat{R}_{A m n} \hat{R}_{A^{\prime} n m} \hat{\varrho}-\hat{R}_{A^{\prime} n m} \hat{\varrho} \hat{R}_{A m n}\right)\right. \\
& + \text { H.c. }],
\end{aligned}
$$

where the primed sum $\Sigma_{A, A^{\prime}}^{\prime}$ indicates that $A \neq A^{\prime}$ and the primed sum $\sum_{m, n}^{\prime}$ indicates that transitions that can strongly interact with the body-assisted electromagnetic field are excluded. In Eq. (5)

$$
\begin{aligned}
\hat{\tilde{H}}_{S}= & \int d^{3} \mathbf{r} \int_{0}^{\infty} d \omega \hbar \omega \hat{\mathbf{f}}^{\dagger}(\mathbf{r}, \omega) \hat{\mathbf{f}}(\mathbf{r}, \omega)+\sum_{A} \sum_{m} \hbar \widetilde{\omega}_{A m} \hat{R}_{A m m} \\
& -\sum_{A} \int_{0}^{\infty} d \omega\left[\hat{\mathbf{d}}_{A} \hat{\hat{\mathbf{E}}}\left(\mathbf{r}_{A}, \omega\right)+\text { H.c. }\right],
\end{aligned}
$$

where 


$$
\widetilde{\omega}_{A m}=\omega_{A m}-\delta_{A A}^{m}, \quad \delta_{A A}^{n}=\sum_{n} \delta_{A A}^{m n}
$$

with $\delta_{A A}^{m n}$ being obtained from

$$
\delta_{A A^{\prime}}^{m n}=\frac{1}{\hbar \pi \varepsilon_{0} c^{2}} \mathcal{P} \int_{0}^{\infty} d \omega \omega^{2} \frac{\mathbf{d}_{A m n} \operatorname{Im} \boldsymbol{G}\left(\mathbf{r}_{A}, \mathbf{r}_{A^{\prime}}, \omega\right) \mathbf{d}_{A^{\prime} m n}^{*}}{\omega-\widetilde{\omega}_{A^{\prime} m n}}
$$

( $\mathcal{P}$ is the principal part) for $A=A^{\prime}$. For $A \neq A^{\prime}$, the parameters $\delta_{A A^{\prime}}^{m n}$ are the dipole-dipole coupling strengths between different atoms $A$ and $A^{\prime}$. Further, the decay rates $\Gamma_{A A^{\prime}}^{m n}$ are defined according to

$$
\Gamma_{A A^{\prime}}^{m n}=\frac{2 \widetilde{\omega}_{A^{\prime} m n}^{2}}{\hbar \varepsilon_{0} c^{2}} \Theta\left(\widetilde{\omega}_{A^{\prime} m n}\right) \mathbf{d}_{A m n} \operatorname{Im} \boldsymbol{G}\left(\mathbf{r}_{A}, \mathbf{r}_{A^{\prime}}, \widetilde{\omega}_{A^{\prime} m n}\right) \mathbf{d}_{A^{\prime} m n}^{*}
$$

$[\Theta(x)$ is the unit step function]. The primed integrals in Eq. (6) indicate that the part of the field that weakly interacts with the atoms has to be omitted, because its effect is taken into account by the level shifting and broadening according to Eqs. (8) and (9).

If the conditions

$$
\begin{aligned}
& \delta_{A A^{\prime}}^{m n}=\delta_{A^{\prime} A}^{m n}, \\
& \Gamma_{A A^{\prime}}^{m n}=\Gamma_{A^{\prime} A}^{m n}
\end{aligned}
$$

are satisfied, which is the case when, for example, the atoms are identical and located in free space or at equivalent positions with respect to the macroscopic bodies, then the master equation (5) takes the somewhat simpler form of

$$
\begin{aligned}
\dot{\hat{\varrho}}= & -\frac{i}{\hbar}\left[\hat{\tilde{H}}_{S}+\hat{H}_{D}, \hat{\varrho}\right]-\frac{1}{2} \sum_{A, A^{\prime}} \sum_{m, n}{ }^{\prime} \Gamma_{A A^{\prime}}^{m n}\left(\hat{R}_{A m n} \hat{R}_{A^{\prime} n m} \hat{\varrho}\right. \\
& \left.-2 \hat{R}_{A^{\prime} n m} \hat{\varrho} \hat{R}_{A m n}+\hat{\varrho} \hat{R}_{A m n} \hat{R}_{A^{\prime} n m}\right),
\end{aligned}
$$

where

$$
\hat{H}_{D}=-\sum_{A, A^{\prime}}, \sum_{m>n} \hbar \Delta_{A A^{\prime}}^{m n}, \hat{R}_{A m n} \hat{R}_{A^{\prime} n m}
$$

describes the dipole-dipole interaction between the atoms, with

$$
\Delta_{A A^{\prime}}^{m n}=\delta_{A A^{\prime}}^{m n}+\delta_{A^{\prime} A}^{n m} .
$$

Equation (12) reveals that the (undamped) system is governed by an effective Hamiltonian equal to $\hat{\widetilde{H}}_{S}+\hat{H}_{D}$. Note that this is not true in general, but only under the conditions (10) and (11).

To construct the (formal) solution to the master equation (12), we first rewrite it in the form of

$$
\dot{\hat{\varrho}}=\hat{L} \hat{\varrho}+\hat{S} \hat{\varrho}
$$

where $\hat{L}$ and $\hat{S}$ are superoperators which act on $\hat{\varrho}$ according to the rules

$$
\hat{L} \hat{\varrho} \equiv-\frac{i}{\hbar}\left(\hat{\mathcal{H}} \hat{\varrho}-\hat{\varrho} \hat{\mathcal{H}}^{\dagger}\right)
$$

$$
\hat{S} \hat{\varrho} \equiv \sum_{A, A^{\prime}} \sum_{m, n}^{\prime} \Gamma_{A A^{\prime}}^{m n} \hat{R}_{A^{\prime} n m} \hat{\varrho} \hat{R}_{A m n},
$$

and the non-Hermitian "Hamiltonian" $\hat{\mathcal{H}}$ reads

$$
\hat{\mathcal{H}}=\hat{\widetilde{H}}_{S}+\hat{H}_{D}-\frac{i \hbar}{2} \sum_{A, A^{\prime}} \sum_{m>n}{ }^{\prime} \Gamma_{A A^{\prime}}^{m n} \hat{R}_{A m n} \hat{R}_{A^{\prime} n m} .
$$

From Eqs. (15)-(17) it then follows that

$$
\hat{\varrho}(t)=\sum_{n=0}^{\infty} \hat{\varrho}^{(n)}(t)
$$

where

$$
\begin{gathered}
\hat{\varrho}^{(0)}(t)=e^{\hat{L}\left(t-t_{0}\right)} \hat{\varrho}\left(t_{0}\right), \\
\hat{\varrho}^{(n)}(t)=\int_{t_{0}}^{t} d t_{n} \int_{t_{0}}^{t_{n}} d t_{n-1} \cdots \int_{t_{0}}^{t_{2}} d t_{1} e^{\hat{L}\left(t-t_{n}\right)} \\
\times \hat{S} e^{\hat{L}\left(t_{n}-t_{n-1}\right)} \cdots \hat{S} e^{\hat{L}\left(t_{1}-t_{0}\right)} \hat{\varrho}\left(t_{0}\right), \quad n=1,2,3 \ldots
\end{gathered}
$$

Although Eq. (19) is not a perturbative expansion, it can be helpful, as we shall see below, in finding the explicit solutions to the master equation.

\section{TWO THREE-LEVEL ATOMS OF $\Lambda$ TYPE}

\section{A. Solution to the master equation}

Let us specify the atomic system and consider two identical three-level atoms $A$ and $B$ of $\Lambda$ type as sketched in Fig. 1. We assume that the dipole-allowed transition $|1\rangle \leftrightarrow|3\rangle$ is tuned to a body-induced electromagnetic field resonance, thereby giving rise to a strong dipole-allowed atom-field coupling. Further, we assume that the dipole-allowed transition $|2\rangle \leftrightarrow|3\rangle$ is weakly coupled to the body-assisted electromagnetic field, and the transition between the states $|1\rangle$ and $|2\rangle$ is dipole forbidden. Restricting our attention to two atoms at equivalent positions with respect to the macroscopic bodies, so that the corresponding transition frequencies are equally shifted and the relations (10) and (11) hold, we may apply the master equation in the form of Eq. (12) and its solution in the form of Eqs. (19)-(21).

Let us assume that the two atoms are initially in the ground state $|1,1\rangle\left(|i, j\rangle \equiv|i\rangle_{A} \otimes|j\rangle_{B}, i, j=1,2,3\right)$ and the rest of the system is prepared in the state

$$
|F\rangle=\int_{0}^{1 \infty} d \omega \int d^{3} \mathbf{r} \mathbf{C}\left(\mathbf{r}, \omega, t_{0}\right) \hat{\mathbf{f}}^{\dagger}(\mathbf{r}, \omega)|\{0\}\rangle,
$$

where $\mathbf{C}\left(\mathbf{r}, \omega, t_{0}\right)$ as a function of $\omega$ is nonzero in a small interval around $\omega \simeq \widetilde{\omega}_{A 31}=\widetilde{\omega}_{B 31}$, and $|\{0\}\rangle$ is the vacuum state. The initial density operator can then be given in the form of $\left(t_{0}=0\right)$ 


$$
\hat{\varrho}(0)=|\Psi(0)\rangle\langle\Psi(0)|, \quad| \Psi(0)\rangle=|1,1\rangle \otimes|F\rangle .
$$

In order to determine the density operator at time $t$, we begin by calculating the first term of the series (19), viz.,

$$
\hat{\varrho}^{(0)}(t)=e^{\hat{L} t} \hat{\varrho}(0)=|\Psi(t)\rangle\langle\Psi(t)| .
$$

Recalling the initial condition (23) and the form of $\hat{\mathcal{H}}$, we may expand $|\Psi(t)\rangle=e^{-i \hat{\mathcal{H}} t / \hbar}|\Psi(0)\rangle$ in rotating-wave approximation as

$$
\begin{aligned}
|\Psi(t)\rangle= & C_{+}(t) e^{-i\left(\widetilde{\omega}_{A 1}+\widetilde{\omega}_{B 3}\right) t}\left|+_{13}\right\rangle \otimes|\{0\}\rangle+C_{-}(t) e^{-i\left(\widetilde{\omega}_{A 1}+\widetilde{\omega}_{B 3}\right) t} \\
& \times\left|-_{13}\right\rangle \otimes|\{0\}\rangle+\int_{0}^{\infty} d \omega \int d^{3} \mathbf{r} e^{-i\left(\widetilde{\omega}_{A 1}+\widetilde{\omega}_{B 1}+\omega\right) t} \\
& \times \mathbf{C}(\mathbf{r}, \omega, t) \hat{\mathbf{f}}^{\dagger}(\mathbf{r}, \omega)|\{0\}\rangle \otimes|1,1\rangle,
\end{aligned}
$$

where $\left| \pm_{13}\right\rangle=2^{-1 / 2}(|3,1\rangle \pm|1,3\rangle)$. Modeling the field resonance strongly coupled to the atomic transition $|1\rangle \leftrightarrow|3\rangle$ by a Lorentzian, with $\omega_{C} \approx \widetilde{\omega}_{A 31}$ and $\Delta \omega_{C}$ being the central frequency and the half width at half maximum, respectively, from the Schrödinger-type equation for $|\Psi(t)\rangle$, we find that $C_{ \pm}(t)$ satisfies the second-order differential equation

$$
\ddot{C}_{ \pm}+a_{ \pm} \dot{C}_{ \pm}+b_{ \pm} C_{ \pm}=\dot{F}_{ \pm}(t)+\left(i \Delta+\Delta \omega_{C}\right) F_{ \pm}(t) .
$$

Here,

$$
\begin{gathered}
a_{ \pm}=i\left(\Delta \mp \Delta_{A B}^{31}\right)+\Delta \omega_{C}+\frac{1}{2} \Gamma^{32}, \\
b_{ \pm}=g_{ \pm}^{2}+\left(\Delta-i \Delta \omega_{C}\right)\left( \pm \Delta_{A B}^{31}+\frac{i}{2} \Gamma^{32}\right) \\
\left(\Gamma^{32} \equiv \Gamma_{A A}^{32}=\Gamma_{B B}^{32}\right), \\
g_{ \pm}^{2}=\frac{1}{2} \Gamma_{ \pm}^{31} \Delta \omega_{C}, \quad \Gamma_{ \pm}^{31}=\Gamma^{31} \pm \Gamma_{A B}^{31}, \\
\Delta=\omega_{C}-\widetilde{\omega}_{A 31}, \text { and } \\
F_{ \pm}(t)=\frac{i}{\hbar \sqrt{2}} \int_{0}^{\infty} d \omega \int d^{3} \mathbf{r} e^{-i\left(\omega-\widetilde{\omega}_{A 31}\right) t} \\
\times \mathbf{d}_{A 31}\left[\widetilde{\boldsymbol{G}}\left(\mathbf{r}_{A}, \mathbf{r}, \omega\right) \pm \widetilde{\boldsymbol{G}}\left(\mathbf{r}_{B}, \mathbf{r}, \omega\right)\right] \mathbf{C}(\mathbf{r}, \omega, 0) .
\end{gathered}
$$

In Eq. (29) $\Gamma^{31} \equiv \Gamma_{A A}^{31}=\Gamma_{B B}^{31}$ and $\Gamma_{A B}^{31}$ are defind according to Eq. (9), but with $\widetilde{\omega}_{A 31}$ being replaced by $\omega_{C}$. Since $\mathbf{C}(\mathbf{r}, \omega, t)$ can be obtained from $C_{ \pm}(t)$, once $C_{ \pm}(t)$ are known, $|\Psi(t)\rangle$ and $\hat{\varrho}^{(0)}(t)=|\Psi(t)\rangle\langle\Psi(t)|$ are known as well.

To calculate the terms $\hat{\varrho}^{(n)}(t)(n>0)$, Eq. (21), of the series (19), we note that the action of the operator $\hat{S}$ on $\hat{\varrho}^{(0)}(t)=|\Psi(t)\rangle\langle\Psi(t)|$ corresponds to atomic transitions $|3\rangle$ $\rightarrow|2\rangle$. Thus, only the states $\left| \pm_{13}\right\rangle$ can contribute to $\hat{S}[|\Psi(t)\rangle\langle\Psi(t)|]$, and we derive

$$
\begin{aligned}
\hat{S}[|\Psi(t)\rangle\langle\Psi(t)|]= & |\{0\}\rangle\langle\{0\}| \otimes\left\{\left(\frac{1}{2} \Gamma_{+}^{32}\left|C_{+}\right|^{2}+\frac{1}{2} \Gamma_{-}^{32}\left|C_{-}\right|^{2}\right)\right. \\
& \times\left|+_{12}\right\rangle\left\langle+_{12}\right|+\left(\frac{1}{2} \Gamma_{+}^{32}\left|C_{-}\right|^{2}+\frac{1}{2} \Gamma_{-}^{32}\left|C_{+}\right|^{2}\right) \\
& \times\left|-_{12}\right\rangle\left\langle-{ }_{12}\right|+\left[\left(\frac{1}{2} \Gamma_{+}^{32} C_{+} C_{-}^{*}+\frac{1}{2} \Gamma_{-}^{32} C_{+}^{*} C_{-}\right)\right. \\
& \left.\left.\times\left|+_{12}\right\rangle\left\langle-{ }_{12}\right|+\text { H.c. }\right]\right\}, \\
& \hat{S} \hat{S}[|\Psi(t)\rangle\langle\Psi(t)|]=0
\end{aligned}
$$

$\left[\Gamma_{ \pm}^{32}=\Gamma^{32} \pm \Gamma_{A B}^{32},\left| \pm_{12}\right\rangle=2^{-1 / 2}(|2,1\rangle \pm|1,2\rangle)\right]$. Recalling that $\hat{\mathcal{H}}$ acts on atomic states in the subspace spanned by $\left| \pm_{13}\right\rangle$, we see that

$$
e^{\hat{L}\left(t-t_{1}\right)} \hat{S}\left[\left|\Psi\left(t_{1}\right)\right\rangle\left\langle\Psi\left(t_{1}\right)\right|\right]=\hat{S}\left[\left|\Psi\left(t_{1}\right)\right\rangle\left\langle\Psi\left(t_{1}\right)\right|\right],
$$

leading to

$$
\varrho^{(1)}(t)=\int_{0}^{t} d t_{1} \hat{S}\left[\left|\Psi\left(t_{1}\right)\right\rangle\left\langle\Psi\left(t_{1}\right)\right|\right]
$$

[cf. Eq. (21)]. Further, Eqs. (32) and (33) imply that $\hat{\varrho}^{(n)}$ $=0$ if $n \geqslant 2$. Thus, the solution to the master equation reads

$$
\hat{\varrho}(t)=|\Psi(t)\rangle\langle\Psi(t)|+\int_{0}^{t} d t_{1} \hat{S}\left[\left|\Psi\left(t_{1}\right)\right\rangle\left\langle\Psi\left(t_{1}\right)\right|\right]
$$

together with Eqs. (25) and (31).

\section{B. Stationary limit}

Let us restrict our attention to the stationary limit $t \rightarrow \infty$. Since $F_{ \pm}(t)$ approaches zero as $t$ tends to infinity, it follows that $\lim _{t \rightarrow \infty} C_{ \pm}(t)=0$. Inserting Eq. (25) in Eq. (35) and taking the trace with respect to the $\hat{\mathbf{f}}$ fields, we derive

$$
\begin{aligned}
\hat{\varrho}_{\mathrm{at}} \equiv & \operatorname{Tr}_{\mathrm{f}} \hat{\varrho}(t \rightarrow \infty) \\
= & \alpha_{+}\left|+{ }_{12}\right\rangle\left\langle+_{12}\left|+\alpha_{-}\right|-_{12}\right\rangle\left\langle-_{12}\right|+\left(\beta\left|+_{12}\right\rangle\left\langle-_{12}\right|+\text { H. . . }\right) \\
& +\left(1-\alpha_{+}-\alpha_{-}\right)|1,1\rangle\langle 1,1|,
\end{aligned}
$$

where

$$
\begin{aligned}
& \alpha_{ \pm}=\frac{1}{2} \int_{0}^{\infty} d t\left(\Gamma_{ \pm}^{32}\left|C_{+}\right|^{2}+\Gamma_{\mp}^{32}\left|C_{-}\right|^{2}\right), \\
& \beta=\frac{1}{2} \int_{0}^{\infty} d t\left(\Gamma_{+}^{32} C_{+} C_{-}^{*}+\Gamma_{-}^{32} C_{+}^{*} C_{-}\right) .
\end{aligned}
$$

To determine the accessible entanglement of the two atoms, it may be instructive to study the concurrence of the atomic subsystem

$$
\mathcal{C}=\sqrt{\lambda_{+}}-\sqrt{\lambda_{-}},
$$

where $\lambda_{ \pm}$are the two eigenvalues $\lambda_{ \pm}$of $\hat{\rho}_{\text {at }} \hat{\tilde{\rho}}_{\text {at }}$, with $\hat{\tilde{\rho}}_{\text {at }}$ being the spin-flipped density operator [30]. A somewhat lengthy but straightforward calculation yields 


$$
\begin{aligned}
\lambda_{ \pm}= & \frac{1}{2}\left\{\alpha_{+}^{2}+\alpha_{-}^{2}-2\left[(\operatorname{Re} \beta)^{2}-(\operatorname{Im} \beta)^{2}\right]\right\} \\
& \pm \frac{1}{2} \sqrt{\left[\left(\alpha_{+}+\alpha_{-}\right)^{2}-4(\operatorname{Re} \beta)^{2}\right]\left[\left(\alpha_{+}-\alpha_{-}\right)^{2}+4(\operatorname{Im} \beta)^{2}\right]}
\end{aligned}
$$

The nearer 1 the value of $\mathcal{C}$ is $(0 \leqslant \mathcal{C} \leqslant 1)$, the higher is the degree of entanglement. Equations (39) and (40) reveal that a noticeably entangled state of the two atoms can be generated if

$$
\alpha_{+}\left(\alpha_{-}\right) \gg \alpha_{-}\left(\alpha_{+}\right),|\beta| ;
$$

thus $\mathcal{C} \rightarrow \alpha_{+}\left(\alpha_{-}\right)$. Needless to say, the entanglement condition (41) is already expected from inspection of Eq. (36).

\section{Different coupling regimes}

Let us return to Eq. (26) and focus on the case where

$$
\dot{F}_{ \pm}(t) \simeq-\left(i \Delta+\Delta \omega_{C}\right) F_{ \pm}(t)
$$

is valid, so that the term on the right-hand side in Eq. (26) can be omitted. Obviously, this is the case when initially the (Lorentzian) field resonance of mid-frequency $\omega_{C}$ and width $\Delta \omega_{C}$ is excited (for details, see Sec. III D). Under the initial conditions $C_{ \pm}(0)=0$ and $\dot{C}_{ \pm}(0)=F_{ \pm}(0)$, the solution to Eq. (26) can then be written in the form of

$$
C_{ \pm}(t)=\frac{F_{ \pm}(0)}{q_{ \pm}} e^{-a_{ \pm} t / 2}\left(e^{q_{ \pm} t / 2}-e^{-q_{ \pm} t / 2}\right)
$$

$\left(q_{ \pm}=\sqrt{a_{ \pm}^{2}-4 b_{ \pm}}\right)$. Restricting again our attention to the stationary limit, we further assume, for simplicity, that both the detuning $\Delta$ and the dipole-dipole coupling strength $\Delta_{A B}^{31}$ vanish. Since even under these conditions the explicit form of the expansion coefficients $\alpha_{ \pm}$, Eq. (37), and $\beta$, Eq. (38), is rather involved, we renounce its presentation here but consider instead some instructive special cases.

From Eqs. (27) and (43) it is seen that the damping constant of $C_{ \pm}$is determined by the sum of the half width at half maximum of the field resonance strongly coupled to the transition $|3\rangle \leftrightarrow|1\rangle$ and the half width at half maximum of the transition $|3\rangle \rightarrow|2\rangle, \Delta \omega_{C}$ and $\Gamma^{32} / 2$, respectively. Due to the finite $\Delta \omega_{C}$, an atom tends to occupy the state $|1\rangle$, while the effect of the finite $\Gamma^{32}$ is that the atom prefers to occupy the state $|2\rangle$. We may therefore restrict ourselves to situations in which

$$
\Gamma^{32} \gg \Delta \omega_{C} .
$$

To achieve noticeable entanglement, the interatomic coupling should be sufficiently strong, i.e., $\left|\Gamma_{A B}^{31}\right| \rightarrow \Gamma^{31}$ and $\left|\Gamma_{A B}^{32}\right| \rightarrow \Gamma^{32}$, equivalently,

$$
\frac{\Gamma_{ \pm}^{31}}{\Gamma_{\bar{\mp}}^{31}} \gg 1, \quad \frac{\Gamma_{ \pm(\mp)}^{32}}{\Gamma_{\bar{\mp}( \pm)}^{32}} \gg 1,
$$

where the upper sign conventions belong together and the lower sign conventions belong together. Note that the first inequality is equivalent to $g_{ \pm} \gg g_{\mp}[$ cf. Eq. (29)]. We now distinguish between the following three cases.

(a) $g_{ \pm} \gg \Gamma^{32} \gg \Delta \omega_{C} \gg g_{\mp}$. In this case, either the symmetric state $\left|+{ }_{13}\right\rangle$ or the antisymmetric state $\left|-_{13}\right\rangle$ is strongly coupled to the medium-assisted electromagnetic field whereas the other one is weakly coupled. For the strongly and weakly coupled states, respectively, Eq. (43) approximates to

$$
C_{ \pm}(t)=\frac{F_{ \pm}(0)}{g_{ \pm}} e^{-\Gamma^{32} t / 4} \sin \left(g_{ \pm} t\right)
$$

and

$$
C_{\mp}(t)=\frac{2 F_{\mp}(0)}{\Gamma^{32}}\left[e^{-\Delta \omega C^{t}}-e^{-\Gamma^{32} t / 2}\right] .
$$

It is seen that $C_{ \pm}(t)$ undergoes damped Rabi oscillations of frequency $g_{ \pm}$, while $C_{\mp}(t)$ undergoes a two-channel exponential decay. The steady-state density operator parameters $\alpha_{ \pm}$, Eq. (37), and $\beta$, Eq. (38), approximate to

$$
\begin{gathered}
\alpha_{ \pm}=\frac{1}{2} \Gamma_{ \pm(\mp)}^{32} \frac{\left|F_{+(-)}(0)\right|^{2}}{g_{+(-)}^{2} \Gamma^{32}}+\Gamma_{\mp( \pm)}^{32} \frac{\left|F_{-(+)}(0)\right|^{2}}{\left(\Gamma^{32}\right)^{2} \Delta \omega_{C}}, \\
\beta=\left[\Gamma_{+}^{32} F_{+}(0) F_{-}^{*}(0)+\Gamma_{-}^{32} F_{+}^{*}(0) F_{-}(0)\right] \frac{\Gamma^{32}}{2 g_{+(-)}^{4}}
\end{gathered}
$$

for $g_{+(-)} \gg g_{-(+)}$.

(b) $g_{ \pm} \gg g_{\mp} \gg \Gamma^{32} \gg \Delta \omega_{C}$. When both $g_{ \pm}$and $g_{\mp}$ dominate the other parameters, then the states $\left|+{ }_{13}\right\rangle$ and $\left|-{ }_{13}\right\rangle$ are both strongly coupled to the medium-assisted electromagnetic field, and Eq. (43) approximates to

$$
C_{ \pm}(t)=\frac{F_{ \pm}(0)}{g_{ \pm}} e^{-\Gamma^{32} t / 4} \sin \left(g_{ \pm} t\right),
$$

which is exactly analogous to Eq. (46). The steady-state density operator parameters $\alpha_{ \pm}$and $\beta$ take the approximate form of

$$
\alpha_{ \pm}=\frac{1}{2} \Gamma_{ \pm}^{32} \frac{\left|F_{+}(0)\right|^{2}}{g_{+}^{2} \Gamma^{32}}+\frac{1}{2} \Gamma_{\overline{+}}^{32} \frac{\left|F_{-}(0)\right|^{2}}{g_{-}^{2} \Gamma^{32}}
$$

and, for $g_{+(-)} \gg g_{-(+)}$,

$$
\beta=\left[\Gamma_{+}^{32} F_{+}(0) F_{-}^{*}(0)+\Gamma_{-}^{32} F_{+}^{*}(0) F_{-}(0)\right] \frac{\Gamma^{32}}{2 g_{+(-)}^{4}} .
$$

(c) $\Gamma^{32} \gg g_{ \pm} \gg g_{\mp}, \Delta \omega_{C}$. When the value of $\Gamma^{32}$ sufficiently exceeds the values of the other parameters, then from Eq. (43) it follows that

$$
C_{ \pm}(t)=\frac{2 F_{ \pm}(0)}{\Gamma^{32}}\left[e^{-\Delta \omega_{C} t}-e^{-\Gamma^{32} t / 2}\right]
$$

i.e., the behavior typical of weakly coupled states is observed [cf. Eq. (47)]. In this approximation, the steady-state density operator parameters $\alpha_{ \pm}$and $\beta$ read 


$$
\alpha_{ \pm}=\Gamma_{ \pm}^{32} \frac{\left|F_{+}(0)\right|^{2}}{\left(\Gamma^{32}\right)^{2}\left(\Delta \omega_{C}+2 g_{+}^{2} / \Gamma^{32}\right)}+\Gamma_{+}^{32} \frac{\left|F_{-}(0)\right|^{2}}{\left(\Gamma^{32}\right)^{2}\left(\Delta \omega_{C}+2 g_{-}^{2} / \Gamma^{32}\right)}
$$

and, for $g_{+(-)} \gg g_{-(+)}$,

$$
\beta=\frac{\Gamma_{+}^{32} F_{+}(0) F_{-}^{*}(0)+\Gamma_{-}^{32} F_{+}^{*}(0) F_{-}(0)}{\left(\Gamma^{32}\right)^{2}\left(\Delta \omega_{C}+g_{+(-)}^{2} / \Gamma^{32}\right)} .
$$

\section{Preparation of the initial state}

One possible way to initially prepare the medium-assisted electromagnetic field in the desired quantum state (22) is to use an additional atom, say atom $D$, such that $\widetilde{\omega}_{D 31}=\widetilde{\omega}_{A 31}$ $=\tilde{\omega}_{B 31}=\omega_{C}$. Let the transition $|1\rangle \leftrightarrow|3\rangle$ of atom $D$ strongly interact with the medium-assisted electromagnetic field in the absence of atoms $A$ and $B$. When atom $D$ is initially prepared in the excited state and the medium-assisted electromagnetic field is initially prepared in the vacuum state, then the probability amplitude of finding, after some interaction time $\Delta t$, atom $D$ (regarded as an effective two-level system) in the ground state and the $\hat{\mathbf{f}}$ fields in a singlequantum state is [29]

$$
\mathbf{C}(\mathbf{r}, \omega, t=0)=\frac{i}{\hbar} \int_{-\Delta t}^{0} d t^{\prime} \mathbf{d}_{D 31}^{*} \widetilde{\boldsymbol{G}}^{*}\left(\mathbf{r}_{D}, \mathbf{r}, \omega\right) e^{i\left(\omega-\widetilde{\omega}_{D 31}\right) t^{\prime}} C_{U_{D}}\left(t^{\prime}\right),
$$

where

$$
C_{U_{D}}(t)=e^{-\Delta \omega_{C}(t+\Delta t) / 2} \cos \left[g_{D}(t+\Delta t)\right]
$$

is the probability amplitude of finding the atom in the upper state. Here,

$$
g_{D}=\sqrt{\Gamma_{D D}^{31} \Delta \omega_{C} / 2}
$$

is the single-atom Rabi frequency. It is seen that $F_{ \pm}(t)$, Eq. (30), calculated by using Eq. (56) satisfies Eq. (42). To calculate $F_{ \pm}(0)$, we fix the interaction time $\Delta t$ such that $C_{U_{D}}(0)=0 ;$ thus

$$
\Delta t=\frac{\pi}{2 g_{D}} .
$$

Combining Eq. (30) with Eqs. (56)-(59), we derive, on applying the Lorentz approximation to the field resonance,

$$
F_{ \pm}(0)=-\frac{\left(\Gamma_{A D}^{31} \pm \Gamma_{B D}^{31}\right) \Delta \omega_{C}}{2 \sqrt{2} g_{D}} \exp \left(-\Delta \omega_{C} \frac{\pi}{2 g_{D}}\right) .
$$

Note that the definition of $\Gamma_{A D}^{31}, \Gamma_{B D}^{31}$, and $\Gamma_{D D}^{31}$ is analogous to that in Sec. III A.

In Eq. (60), the exponential factor characterizes the photon loss during the interaction time due to the finite width of the field resonance. Obviously, the better the strong-coupling condition $\Delta \omega_{C} \ll g_{D}$ is satisfied, the less the photon loss is. For example, when atom $A$ changes places with atom $D$ and the orientations of the transition dipole moments of atoms $A$ and $D$ are the same, then from Eq. (60) [together with Eq. (29)] it follows that $\left(\Delta \omega_{C} \ll g_{D}\right)$

$$
F_{ \pm}(0) \simeq-\frac{g_{ \pm}^{2}}{\sqrt{\left(\Gamma_{+}^{31}+\Gamma_{-}^{31}\right) \Delta \omega_{C} / 2}}
$$

thus $F_{+}(0) \simeq-g_{+}$and $F_{-}(0) \simeq-g_{-}^{2} / g_{+}$if $\Gamma_{+}^{31} / \Gamma_{-}^{31} \gg 1$, while $F_{-}(0) \simeq-g_{-}$and $F_{+}(0) \simeq-g_{+}^{2} / g_{-}$if $\Gamma_{-}^{31} / \Gamma_{+}^{31} \gg 1$. When atom $B$ changes places with atom $D$ and the orientations of the transition dipole moments of atoms $B$ and $D$ are the same, then the signs of both $F_{+}(0)$ and $F_{-}(0)$ are reversed [cf. Eq. (60)]. The state preparation is not affected though [cf. Eqs. (37), (38), and (43)]. It is worth noting that, as we will see in Sec. IV, the highest degree of entanglement can be achieved in case of equal positions of atoms $D$ and $A$ (or $B$ ).

\section{ATOMIC ENTANGLEMENT NEAR A DIELECTRIC MICROSPHERE}

Let us apply the theory to two atoms near a dispersing and absorbing dielectric microsphere (of radius $R$ ) characterized by a Drude-Lorentz type permittivity

$$
\varepsilon(\omega)=1+\frac{\omega_{P}^{2}}{\omega_{T}^{2}-\omega^{2}-i \omega \gamma}
$$

( $\omega_{P}$ is the coupling constant; $\omega_{T}$ is the transverse resonance frequency; $\gamma$ is the absorption parameter), which features a band gap in the region $\omega_{T}<\omega<\omega_{L}=\sqrt{\omega_{T}^{2}+\omega_{P}^{2}}$, where $\operatorname{Re} \varepsilon(\omega)<0$.

\section{A. Two-atom coupling}

We first estimate how well the conditions (45) can be satisfied. Making use of the Green tensor as given in Ref. [31], one can show, on assuming radial dipole orientations, that Eq. (9) leads to

$$
\begin{aligned}
\Gamma_{A B}= & \frac{3}{2} \Gamma_{0} \operatorname{Re} \sum_{l=1}^{\infty} \frac{l(l+1)(2 l+1)}{(k r)^{2}} h_{l}^{(1)}(k r) \\
& \times\left[j_{l}(k r)+B_{l}^{N}(\omega) h_{l}^{(1)}(k r)\right] P_{l}(\cos \theta),
\end{aligned}
$$

where $\Gamma_{A B} \equiv \Gamma_{A B}^{m n}, k=\omega / c, \omega \equiv \widetilde{\omega}_{A}^{m n}=\widetilde{\omega}_{B}^{m n}>0$, and $r \equiv r_{A}=r_{B}$. Since the following discussion applies to both the transitions $|3\rangle \leftrightarrow|1\rangle$ and $|3\rangle \leftrightarrow|2\rangle$, we omit the level labels $m$ and $n$. Further, $\Gamma_{0}$ is the corresponding single-atom decay rate in free space, $j_{l}(z)$ and $h_{l}^{(1)}(z)$ are the spherical Bessel and Hankel functions, respectively, $P_{l}(x)$ is the Legendre function, $\theta$ is the angle between the two transition dipole moments $\left(\left|\mathbf{d}_{A}\right|=\left|\mathbf{d}_{B}\right|\right)$, and $B_{l}^{N}(\omega)$ are the scattering coefficients [31]. Note that radially oriented dipoles couple only to TM waves, whereas tangentially oriented dipoles couple to both TM and TE waves (for details, see, e.g., [32]).

The complex roots of the denominator of the reflection coefficients $B_{l}^{N}(\omega)$ determine the positions and the widths of the sphere-assisted electromagnetic field resonances. When $\omega$ coincides with a resonance frequency, say $\omega_{C}$, then the corresponding $l$ term in Eq. (63) is the leading one; thus 


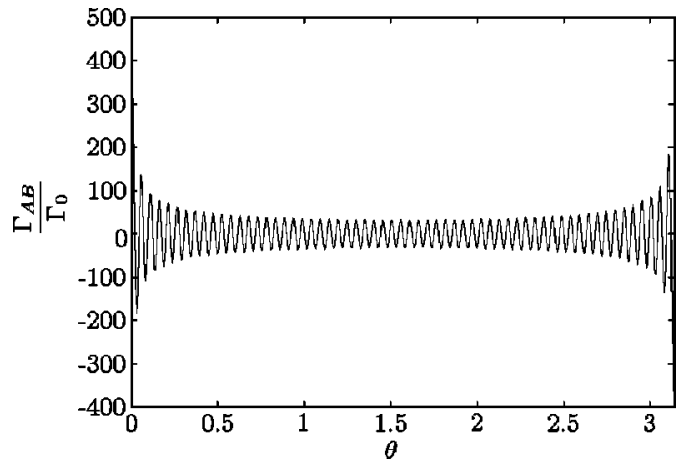

FIG. 2. The two-atom collective decay rate $\Gamma_{A B}$ [Eq. (63)] as a function of the angle $\theta$ between the transition dipole moments for $\omega \simeq 1.0501 \omega_{T}$. The two atoms are at distances $\Delta r \equiv r-R$ $=0.14 \lambda_{T}\left(\lambda_{T}=2 \pi c / \omega_{T}\right)$ from the surface of a dielectric sphere $\left(\omega_{P}\right.$ $\left.=0.5 \omega_{T}, \gamma=10^{-6} \omega_{T}, R=10 \lambda_{T}\right)$.

$$
\begin{aligned}
\Gamma_{A B} \simeq & \frac{3}{2} \Gamma_{0} \operatorname{Re}\left\{\frac{l(l+1)(2 l+1)}{(k r)^{2}} h_{l}^{(1)}(k r)\right. \\
& \left.\times\left[j_{l}(k r)+B_{l}^{N}(\omega) h_{l}^{(1)}(k r)\right] P_{l}(\cos \theta)\right\}
\end{aligned}
$$

$\left(\omega \simeq \omega_{C}\right)$. Equation (64) implies that when the two atoms are at diametrically opposite positions with respect to the sphere, i.e., $\theta=\pi$ and hence $P_{l}(\cos \theta)=(-1)^{l}$, then the interaction of the symmetric (antisymmetric) state with the sphere-assisted electromagnetic field is enhanced, while the antisymmetric (symmetric) state almost decouples [cf. Eq. (29)].

The dependence on $\theta$ of $\Gamma_{A B}$ as given by Eq. (63) is illustrated in Fig. 2, where the atomic transition frequency $\omega$ is chosen to be close to a resonance frequency of the sphereassisted electromagnetic field, namely, the first one on the left in Fig. 3. Note that $\Gamma_{A B} \rightarrow \Gamma \equiv \Gamma_{A A}=\Gamma_{B B}$ for $\theta \rightarrow 0$. From Fig. 3 it is clearly seen that the value of $\Gamma_{+}\left(\Gamma_{-}\right)$can drastically exceed the value of $\Gamma_{-}\left(\Gamma_{+}\right)$when the two atoms approach the microsphere and the transition frequency equals a

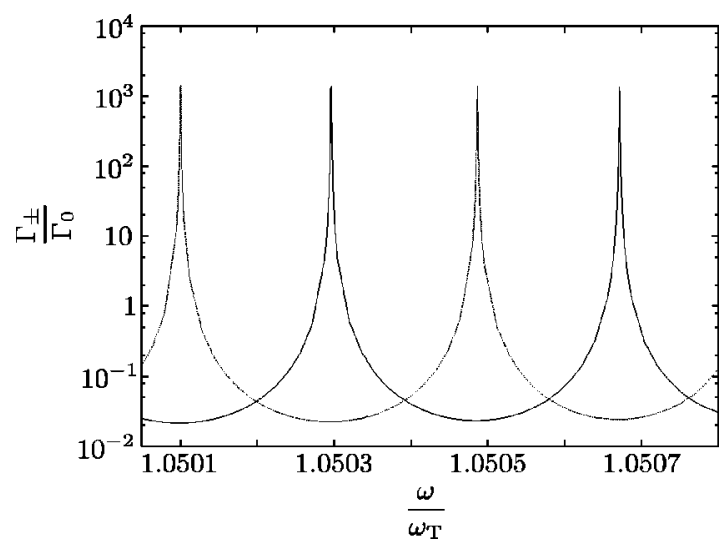

FIG. 3. The two-atom decay rates $\Gamma_{+}=\Gamma+\Gamma_{A B}$ (solid curve) and $\Gamma_{-}=\Gamma-\Gamma_{A B}$ (dotted curve) for the symmetric and antisymmetric states, respectively, as functions of the transition frequency $\omega$, with $\Gamma_{A B}$ from Eq. (63) $\left(\Gamma \equiv \Gamma_{A A}=\lim _{\theta \rightarrow 0} \Gamma_{A B}\right)$. The other parameters are the same as in Fig. 2.

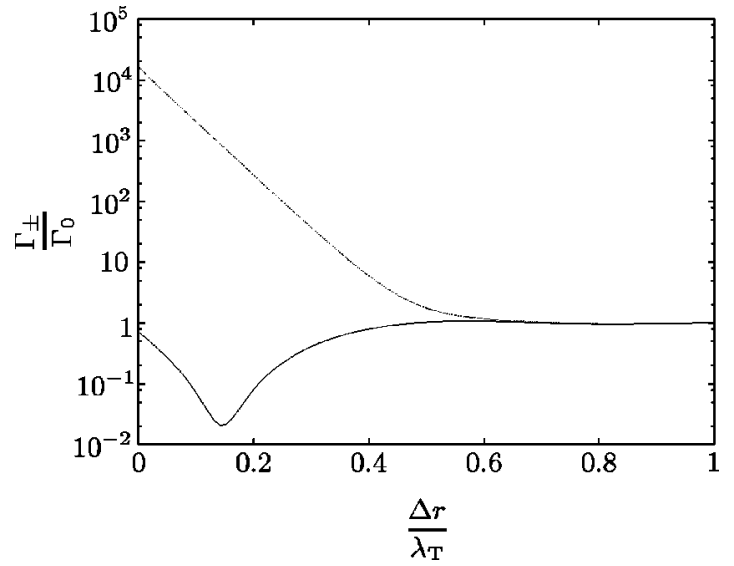

FIG. 4. The two-atom decay rates $\Gamma_{+}=\Gamma+\Gamma_{A B}$ (solid curve) and $\Gamma_{-}=\Gamma-\Gamma_{A B}$ (dotted curve) for the symmetric and antisymmetric states, respectively, as functions of the distance $\Delta r$ between the atom and the surface of the dielectric sphere, with $\Gamma_{A B}$ from Eq. (63) $\left(\Gamma \equiv \Gamma_{A A}=\lim _{\theta \rightarrow 0} \Gamma_{A B}\right)$. The other parameters are the same as in Fig. 2.

resonance frequency of the sphere-assisted electromagnetic field. Recall that $\Gamma_{+}\left(\Gamma_{-}\right)$is a measure of the strength of coupling of the symmetric (antisymmetric) state to the sphereassisted field. Figure 4 reveals that, for chosen resonance frequency of the microsphere, there is an optimum distance-the distance at which the solid curve attains the minimum-for which the best contrast between $\Gamma_{+}$and $\Gamma_{-}$ can be realized. With increasing distance of the atoms from the sphere, the values of both $\Gamma_{+}$and $\Gamma_{-}$tend to the freespace value $\Gamma_{0}$ as they should.

Figures 2-4 refer to atomic transition frequencies within the band gap. In this case, the strong two-atom interaction observed when the atoms are at diametrically opposite positions with respect to the sphere is mediated by SG waves. Of course, the effect of enhanced $\Gamma_{+}\left(\Gamma_{-}\right)$and simultaneously reduced $\Gamma_{-}\left(\Gamma_{+}\right)$can also be observed for transition frequencies below the band gap. In this case, the sphere-assisted field resonances correspond to $\mathrm{WG}$ waves. An example is shown in Fig. 5. Figures 3 and 5 also convey a feeling of the sharpness of the field resonances, which ranges from being very sharp to being less so. The sharpness can be improved by increasing the microsphere radius or by reducing the material absorption. Note that WG waves suffer much more from absorption than do SG waves (see, e.g., Ref. [32]).

\section{B. Entanglement of two $\Lambda$-type atoms}

The results given in Sec. IV A show that the optimal positions of two $\Lambda$-type atoms $A$ and $B$, which are desired to entangle with each other near a microsphere, are diametrically opposite with respect to the sphere. Further, the transition frequency $\widetilde{\omega}_{A 31}=\widetilde{\omega}_{B 31}$ should coincide with the (mid-)frequency $\omega_{C}$ of a sufficiently sharply peaked sphereassisted electromagnetic field resonance, so that the strongcoupling regime is realized and the first of the conditions (45) is satisfied. Finally, the transition frequency $\widetilde{\omega}_{A 32}$ $=\widetilde{\omega}_{B 32}$ should coincide with the (mid-)frequency of some 


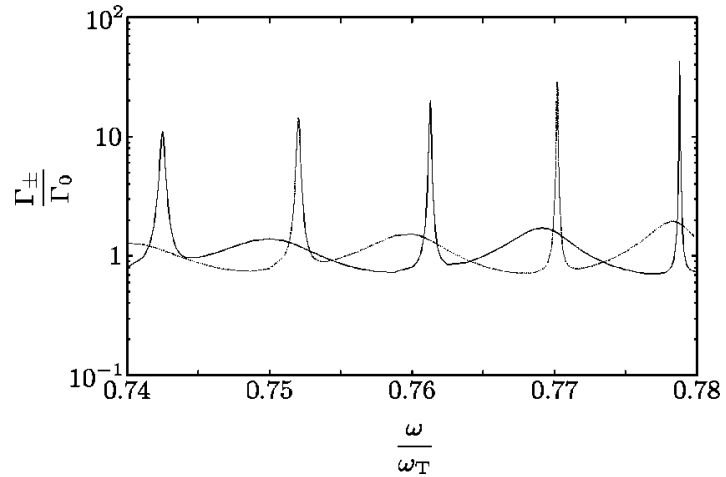

FIG. 5. The two-atom decay rates $\Gamma_{+}=\Gamma+\Gamma_{A B}$ (solid curve) and $\Gamma_{-}=\Gamma-\Gamma_{A B}$ (dotted curve) for the symmetric and antisymmetric states, respectively, as functions of the transition frequency $\omega$, with $\Gamma_{A B}$ from Eq. (63) $\left(\Gamma \equiv \Gamma_{A A}=\lim _{\theta \rightarrow 0} \Gamma_{A B}\right)$. The other parameters are the same as in Fig. 2.

moderately peaked field resonance, so that the second of the conditions (45) is also satisfied, but the weak-coupling regime applies, thereby giving rise to an irreversible decay channel. As a result, the condition (44) can also be expected to be satisfied. By choosing atoms with appropriate transition dipole matrix elements (for more detailed estimations, see Ref. [29]), all the conditions including both the inequalities characterizing the three cases (a)-(c) in Sec. III C and the field-preparation conditions (61) can be satisfied. Let us examine the cases (a)-(c) in more detail.

(a) $g_{ \pm} \gg \Gamma^{32} \gg \Delta \omega_{C} \gg g_{\mp}$. For definiteness, let $\Gamma_{+}^{31} \gg \Gamma_{-}^{31}$ and $\Gamma_{+}^{32} \gg \Gamma_{-}^{32}$. When atom $A$ (or $B$ ) changes places with atom $D$, which provides the initial field excitation, and Eq. (61) applies, then Eqs. (48) and (49) lead to

$$
\begin{aligned}
& \alpha_{+} \simeq 1, \\
& \alpha_{-} \simeq \frac{\Gamma_{-}^{32}}{2 \Gamma^{32}}+\frac{2 g_{-}^{4}}{g_{+}^{2} \Gamma^{32} \Delta \omega_{C}} \ll 1, \quad \beta \simeq\left(\frac{\Gamma^{32} g_{-}}{g_{+}^{2}}\right)^{2} \ll 1 .
\end{aligned}
$$

Hence, an almost perfectly entangled state is produced, $\hat{\varrho}_{\text {at }}$ $\simeq\left|+_{12}\right\rangle\left\langle+_{12}\right|$ [see Eq. (36)], and, accordingly, $\mathcal{C} \simeq 1$ is achieved. Clearly, $\alpha_{+}=1(\mathcal{C}=1)$ cannot be exactly realized, because of the losses unavoidably associated with the always finite width of the field resonance. It is worth mentioning that when the positions of atoms $D$ and $A$ (or $B$ ) are different from each other (e.g., atom $D$ is equidistant from atoms $A$ and $B$ ), then the degree of entanglement that can be achieved is smaller than that in the case of equal positions in general. Note that when $\Gamma_{-}^{31} \gg \Gamma_{+}^{31}$ and $\Gamma_{-}^{32} \gg \Gamma_{+}^{32}$, then $\hat{\varrho}_{a t}$ $\simeq\left|+_{12}\right\rangle\left\langle+_{12}\right|$ is also valid. For $\Gamma_{ \pm}^{31} \gg \Gamma_{\mp}^{31}$ and $\Gamma_{\mp}^{32} \gg \Gamma_{ \pm}^{32}$, however, the roles of $\alpha_{+}$and $\alpha_{-}$are interchanged and $\hat{\varrho}_{\text {at }}$ $\simeq\left|-{ }_{12}\right\rangle\left\langle-{ }_{12}\right|$.

For $\Gamma_{+}^{31} \gg \Gamma_{-}^{31}$ and $\Gamma_{+}^{32} \gg \Gamma_{-}^{32}$, the two-atom system undergoes fast $|1,1\rangle \leftrightarrow\left|{ }_{13}\right\rangle$ Rabi oscillations as long as one of the two atoms jumps to state $|2\rangle$, but we do not know which one. Hence, the result is an entangled state between one atom in the state $|2\rangle$ and the other in the state $|1\rangle$. The time after which the stationary limit is established is determined by the lifetime $\sim\left(\Gamma^{32}\right)^{-1}$ of the short-living state $\left|+_{13}\right\rangle$, while the long-living state $\left|-_{13}\right\rangle$ of lifetime $\sim\left(\Delta \omega_{C}\right)^{-1}$ is practically unpopulated [cf. Eqs. (46) and (47)].

(b) $g_{ \pm} \gg g_{\mp} \gg \Gamma^{32} \gg \Delta \omega_{C}$. For definiteness, let again assume that $\Gamma_{+}^{31} \gg \Gamma_{-}^{31}$ and $\Gamma_{+}^{32} \gg \Gamma_{-}^{32}$. From Eqs. (51) and (52) together with Eq. (61) we obtain

$$
\begin{gathered}
\alpha_{+} \simeq 1, \\
\alpha_{-} \simeq \frac{\Gamma_{-}^{32}}{2 \Gamma^{32}}+\frac{2 g_{-}^{2}}{g_{+}^{2}} \ll 1, \quad \beta \simeq\left(\frac{\Gamma^{32} g_{-}}{g_{+}^{2}}\right)^{2} \ll 1 .
\end{gathered}
$$

Thus, this coupling regime leaves the two atoms in an entangled state analogous to case (a). However, since the inequality $g_{ \pm} \gg g_{\mp}$ requires $g_{ \pm}$to be as large as possible and $g_{\mp}$ to be as small as possible, while the inequality $g_{\mp} \gg \Gamma^{32}$ requires that $g_{\mp}$ is much larger than $\Gamma^{32}$, it may be more difficult to realize this regime. Note that $g_{\mp}$ is the smallest or one of the smallest parameters in the cases (a) and (c).

(c) $\Gamma^{32} \gg g_{ \pm} \gg g_{\mp}, \Delta \omega_{C}$. In this case, the irreversible decay from state $|3\rangle$ to state $|2\rangle$ is so dominant that Rabi oscillations are fully suppressed in the time evolution of both $C_{+}$and $C_{-}$ [see Eq. (53)]. From Eq. (54) we obtain, on making use of Eq. (61) and again assuming $\Gamma_{+}^{31} \gg \Gamma_{-}^{31}$ and $\Gamma_{+}^{32} \gg \Gamma_{-}^{32}$,

$$
\alpha_{+} \simeq \frac{2 g_{+}^{2} / \Gamma^{32}}{\Delta \omega_{C}+2 g_{+}^{2} / \Gamma^{32}} .
$$

To generate the entangled state $\left|+{ }_{12}\right\rangle$, i.e., $\alpha_{+} \simeq 1$, the additional condition

$$
\frac{g_{+}}{\Gamma^{32}} \gg \frac{\Delta \omega_{C}}{g_{+}}
$$

must be required to be satisfied, as can be seen from Eq. (69). The parameters $\alpha_{-}$and $\beta$ then read

$$
\alpha_{-} \simeq \frac{\Gamma_{-}^{32}}{2 \Gamma^{32}}+\frac{g_{-}^{2}}{g_{+}^{2}} \ll 1, \quad \beta \simeq \frac{2 g_{-}^{2}}{g_{+}^{2}} \ll 1 .
$$

In a similar fashion, it can be shown that in case of $\Gamma_{ \pm}^{31}$ $\gg \Gamma_{\bar{\mp}}^{31}$ and $\Gamma_{\bar{\mp}}^{32} \gg \Gamma_{ \pm}^{32}$ the antisymmetric entangled state $\left|-{ }_{12}^{ \pm}\right\rangle$ is generated.

The inequality (70) can be understood as follows. For $F_{+}(0) \simeq-g_{+}$, Eq. (53) yields

$$
C_{+}^{13}(t) \simeq-\left(2 g_{+} / \Gamma^{32}\right)\left[e^{-\Delta \omega} C^{t}-e^{-\Gamma^{32} t / 2}\right],
$$

i.e., $C_{+}^{13}(t) \sim g_{+} / \Gamma^{32}$. Thus, though one can allow for $g_{+} / \Gamma^{32} \ll 1$, this ratio has still to satisfy the inequality (70) such that there is a nonvanishing probability that one of the atoms can reach the state $|3\rangle$ from the initial state $|1\rangle$ to jump to the state $|2\rangle$.

\section{SUMMARY AND CONCLUSIONS}

We have proposed a scheme for nonconditional preparation of two spatially well-separated identical atoms in longliving highly entangled states. The scheme uses $\Lambda$-type atoms passing a resonatorlike equipment of realistic, dispersing, and absorbing macroscopic bodies which form 
electromagnetic field resonances, the heights and widths of which are determined by the radiative and nonradiative (absorption) losses. The lowest-lying atomic state and the lowerlying excited state, which can be the ground state and a metastable state or two metastable states, play the role of the basis states of an atomic qubit. The atoms initially prepared in the lowest-lying states are pumped by a single-excitation "pulse" of the body-assisted electromagnetic field, thereby strongly driving the dipole-allowed transition between the lowest- and highest-lying atomic states. In this way, one of the two atoms-we do not know which one - can absorb the single-photonic excitation, and subsequent irreversible spontaneous decay of the excited atomic state to a lower-lying excited state, the transition of which to the lowest-lying state is dipole forbidden, results in a metastable two-atom entangled state.

To be quite general, we first developed the theory, without specifying the atoms and the equipment whose body-assisted electromagnetic field is used for the the collective atom-field interaction. For the case of two $\Lambda$-type atoms, we have derived the general solution of the coupled field-atom evolution equations and presented special coupling conditions under which high-degree entanglement can be achieved. We have then applied the theory to the problem of entanglement of two $\Lambda$-type atoms near a microsphere. In particular, we have shown that the scheme is capable of realizing strong coupling in one arm and weak coupling in the other arm of the $\Lambda$ configuration. In this context, we have also analyzed the preparation of the initial single-photonic field excitation required for initiating the process of entanglement.

In contrast to the common sense idea that the existence of dissipation spoils the quantum coherence of a system, dissipation is here essential to transfer the entanglement from the strongly driven transitions to the dipole-forbidden transitions. The fact that only ground or metastable states serve as basis states of the qubits guarantees the long lifetime of the entangled state. It is worth noting that the scheme renders it possible to test nonlocality for a two-atom system. The atoms in a pair passing by a microsphere and being entangled there can be separated from each other and one can be sure that in the meantime the entanglement is not lost.

\section{ACKNOWLEDGMENTS}

This work was supported by the Deutsche Forschungsgemeinschaft. Ö.Ç. and H.T.D., respectively, acknowledge support from the Scientific and Technical Research Council of Turkey and the Vietnam National Program for Basic Research.
[1] E. S. Polzik, Phys. Rev. A 59, 4202 (1999).

[2] M. D. Lukin, S. F. Yelin, and M. Fleischhauer, Phys. Rev. Lett. 84, 4232 (2000).

[3] A. Kuzmich and E. S. Polzik, Phys. Rev. Lett. 85, 5639 (2000).

[4] K. Mølmer, Opt. Commun. 179, 429 (2000).

[5] S. Lloyd, M. S. Shahriar, J. H. Shapiro, and P. R. Hemmer, Phys. Rev. Lett. 87, 167903 (2001).

[6] B. Kraus and J. I. Cirac, Phys. Rev. Lett. 92, 013602 (2004).

[7] M. Paternostro, W. Son, and M. S. Kim, Phys. Rev. Lett. 92, 197901 (2004), M. Paternostro, W. Son, M. S. Kim, G. Falci, and G. M. Palma, Phys. Rev. A 70, 022320 (2004).

[8] C. Cabrillo, J. I. Cirac, P. García-Fernández, and P. Zoller, Phys. Rev. A 59, 1025 (1999).

[9] S. Bose, P. L. Knight, M. B. Plenio, and V. Vedral, Phys. Rev. Lett. 83, 5158 (1999).

[10] L. -M. Duan, J. I. Cirac, P. Zoller, and E. S. Polzik, Phys. Rev. Lett. 85, 5643 (2000).

[11] B. Julsgaard, A. Kozhekin, and E. S. Polzik, Nature (London) 413, 400 (2001).

[12] L. -M. Duan, M. D. Lukin, J. I. Cirac, and P. Zoller, Nature (London) 414, 413 (2001).

[13] L. -M. Duan, Phys. Rev. Lett. 88, 170402 (2002).

[14] A. Gilchrist, A. G. White, and W. J. Munro, Phys. Rev. A 66, 012106 (2002).

[15] X. -L. Feng, Z. -M. Zhang, X. -D. Li, S. -Q. Gong, and Z. -Z. Xu, Phys. Rev. Lett. 90, 217902 (2003).

[16] L. -M. Duan and H. J. Kimble, Phys. Rev. Lett. 90, 253601 (2003).

[17] C. Simon and W. T. M. Irvine, Phys. Rev. Lett. 91, 110405
(2003).

[18] D. E. Browne, M. B. Plenio, and S. F. Huelga, Phys. Rev. Lett. 91, 067901 (2003).

[19] S. Clark, A. Peng, M. Gu, and S. Parkins, Phys. Rev. Lett. 91, 177901 (2003).

[20] E. Hagley, X. Maitre, G. Nogues, C. Wunderlich, M. Brune, J. M. Raimond, and S. Haroche, Phys. Rev. Lett. 79, 1 (1997).

[21] A. Rauschenbeutel, G. Nogues, S. Osnaghi, P. Bertet, M. Brune, J. M. Raimond, and S. Haroche, Science 288, 2024 (2000).

[22] S. Osnaghi, P. Bertet, A. Auffeves, P. Maioli, M. Brune, J. M. Raimond, and S. Haroche, Phys. Rev. Lett. 87, 037902 (2001).

[23] Ho Trung Dung, S. Scheel, D. -G. Welsch, and L. Knöll, J. Opt. B: Quantum Semiclassical Opt. 4, 169 (2002).

[24] A. M. Basharov, JETP Lett. 75, 123 (2002).

[25] Z. Ficek and R. Tanaś, J. Mod. Opt. 50, 2765 (2003).

[26] J. Kästel and M. Fleischhauer, e-print quant-ph/0404063.

[27] M. A. Can, Ö. Çakir, A. Klyachko, and A. Shumovsky, Phys. Rev. A 68, 022305 (2003).

[28] L. Knöll, S. Scheel, and D. -G. Welsch, in Coherence and Statistics of Photons, edited by J. Perina (Wiley, New York, 2001), p. 1; for an update, see e-print quant-ph/003121.

[29] Ho Trung Dung, L. Knöll, and D. -G. Welsch, Phys. Rev. A 66, 063810 (2002).

[30] W. K. Wootters, Phys. Rev. Lett. 80, 2245 (1998).

[31] L. W. Li, M. S. Kooi, and T. S. Yeo, IEEE Trans. Microwave Theory Tech. 42, 20302 (1994).

[32] Ho Trung Dung, L. Knöll, and D. -G. Welsch, Phys. Rev. A 64, 013804 (2001). 